\title{
Bibliographie générale de la partie thématique
}

\section{(2) OpenEdition}

1 Journals

Édition électronique

URL : http://journals.openedition.org/austriaca/654

DOI : 10.4000/austriaca.654

ISSN : 2729-0603

Éditeur

Presses universitaires de Rouen et du Havre

\section{Édition imprimée}

Date de publication : 1 juin 2018

Pagination : 225-243

ISBN : 979-10-240-1233-9

ISSN : 0396-4590

\section{Référence électronique}

"Bibliographie générale de la partie thématique », Austriaca [En ligne], 86 | 2018, mis en ligne le 09 juillet 2020, consulté le 30 janvier 2021. URL : http://journals.openedition.org/austriaca/654; DOI : https://doi.org/10.4000/austriaca.654 


\title{
Bibliographie générale de la partie thématique
}

\section{Classée par auteur traité dans ce volume}

\author{
Ludwig Anzengruber
}

Rossbacher Karlheinz, "Ludwig Anzengruber (1839-1889)", Nestroyana, $\mathrm{n}^{\mathrm{o}} 29,2009$, p. 133-143.

Weber Fritz, Anzengrubers Naturalismus, Dissertation, Tübingen, 1928.

Hermann Bahr

\section{CEuvres}

BAHR Hermann, «Die Moderne», Moderne Dichtung, vol. 1, $\mathrm{n}^{0}$ 1, 1890, p. 13-15.

—, Zur Kritik der Moderne, Zürich, Verlags-Magazin, 1890.

-, Die Überwindung des Naturalismus, Dresden und Leipzig, E. Pierson's, 1891.

—, Studien zur Kritik der Moderne, Frankfurt am Main, Rütten \& Loening, 1894.

—, Renaissance, Berlin, S. Fischer, 1897.

—, «Die Entdeckung der Provinz », Neues Wiener Tagblatt, $\mathrm{n}^{\mathrm{o}} 270,1^{\mathrm{er}}$ octobre 1899, p. 1-3.

—, Bildung, Berlin-Leipzig, Insel-Verlage bei Schuster \& Loeffler, 1900.

—, Das Hermann-Bahr-Buch, Berlin, S. Fischer, 1913.

—, Selbstbildnis, Berlin, S. Fischer, 1923.

—, Tagebücher, Skizzenbücher, Notizhefte, vol. 2: 1890-190o, Moritz Csáky (éd.), Wien-Köln-Weimar, Böhlau, 1996.

\section{Critique}

BACHLEITNER Norbert, "Hermann Bahr und die französische Literatur ", dans Johann Lachinger (dir.), Hermann Bahr. Mittler der europäischen Moderne, Linz, Jahrbuch des Adalbert-Stifter-Instituts des Landes Oberösterreich, 2001, p. 145-160. 
Bourget Paul, Études et portraits, Paris, Alphonse Lemerre, 1889, t. I.

Daviau Donald G., Der Mann von Übermorgen. Hermann Bahr 1863-1934, Wien, Böhlau, 1984.

Herzfeld Marie, „Hermann Bahr, ,Die Überwindung des Naturalismus““, Wiener Literatur-Zeitung, vol. 2, $\mathrm{n}^{\mathrm{o}}$ 10, 15 août 1891, p. 10-11.

KAfKA Eduard Michael, "Der neueste Bahr», Moderne Rundschau, vol. 3, $\mathrm{n}^{\mathrm{o}}$ 5-6, 15 juin 1891, p. 220-222.

—, "Zur Kritik der Moderne », Moderne Dichtung, vol. 1, nº 2, $1^{\mathrm{er}}$ février 1890, p. 120-122.

Kindermann Heinz, Hermann Bahr. Ein Leben für das europäische Theater, Graz-Köln, Hermann Böhlaus Nachf., 1954.

Kraus Karl, «Die demolirte Literatur», Wiener Rundschau, vol. 1, $\mathrm{n}^{\mathrm{o}}$, 15 novembre 1896, p. 19-27.

MAyerhofer Lukas, «Facetten einer Rezeption : Hermann Bahr und Henrik Ibsen ", dans Johann Lachinger (dir.), Hermann Bahr. Mittler der europäischen Moderne, Linz, Jahrbuch des Adalbert-Stifter-Instituts des Landes Oberösterreich, 2001, p. 71-86.

Streim Gregor, “ „Die richtige Moderne.“ Hermann Bahr und die Formierung der literarischen Moderne in Berlin », Hofmannsthal-Jahrbuch, $\mathrm{n}^{\circ} 4$, 1996, p. 323-359.

SusEN Gerd-Hermann, “ „Das Alte kracht in allen Fugen!“ Hermann Bahr und die Freie Bühne für modernes Leben », dans Martin Anton Müller, Claus Pias et Gottfried Schnödl (dir.), Hermann Bahr : Österreichscher Kritiker europäischer Avantgarden, Bern, Peter Lang, 2014, p. 39-54.

Tateo Giovanni, «In der Werkstatt der Wiener Moderne. Der Beitrag Hermann Bahrs», dans Mauro Ponzi (dir.), Klassische Moderne. Ein Paradigma des 20. Jahrhundert, Würzburg, Königshausen \& Neumann, 2010, p. 133-149.

—, " "Ganz anders die jungen Wiener” Hermann Bahr e il recente passato letterario austriaco come spazio di transizione al Moderno », dans « Letterature del Danubio ", Cultura tedesca, $n^{\circ}$ 40, janvier-juin 2011, p. 93-104.

ZIEGER Karl, «La "Modernité viennoise" : de la réception du naturalisme à une "mystique des nerfs" », dans Xavier Garnier et Anne Tomiche (dir.), Modernités occidentales et extra-occidentales, Paris, L'Harmattan, "Itinéraires, littérature, textes, cultures, 3 ", 2009, p. 135-149.

\section{Hermann Broch}

\section{CEuvres}

Broch Hermann, Kommentierte Werkausgabe, Paul Michael Lützeler (éd.), Frankfurt am Main, Suhrkamp, 1974-1981. 
-, «James Joyce und die Gegenwart. Rede zu Joyces 50. Geburtstag» [1932], dans Schriften zur Literatur 1: Kritik, Kommentierte Werkausgabe, Paul Michael Lützeler (éd.), Frankfurt am Main, Suhrkamp, 1975, t. IX, vol. 1, p. 63-94.

-, «Philistrosität, Realismus, Idealismus in der Kunst» [1913], dans Schriften zur Literatur 1: Kritik, Kommentierte Werkausgabe, Paul Michael Lützeler (éd.), Frankfurt am Main, Suhrkamp, 1975, t. IX, vol. 1, p. 13-29.

-, «Zolas Vorurteil» [1916], dans Schriften zur Literatur 1: Kritik, Kommentierte Werkausgabe, Paul Michael Lützeler (éd.), Frankfurt am Main, Suhrkamp, 1975, t. IX, vol. 1, p. 34-40.

-, « Das Böse im Wertsystem der Kunst » [1933], dans Schriften zur Literatur 2: Theorie, Kommentierte Werkausgabe, Paul Michael Lützeler (éd.), Frankfurt am Main, Suhrkamp, 1976, t. IX, vol. 2, p. 119-157.

-, "Das Weltbild des Romans. Ein Vortrag" [1933], dans Schriften zur Literatur 2: Theorie, Kommentierte Werkausgabe, Paul Michael Lützeler (éd.), Frankfurt am Main, Suhrkamp, 1976, t. IX, vol. 2, p. 89-118.

-, Die Schlafwandler. Eine Romantrilogie, dans Kommentierte Werkausgabe, Paul Michael Lützeler (éd.), Frankfurt am Main, Suhrkamp, 1978, t. I.

-, «Eine methodologische Novelle» [1918], dans Novellen, Kommentierte Werkausgabe, Paul Michael Lützeler (éd.), Frankfurt am Main, Suhrkamp, 1980, p. 11-23.

\section{Critique}

CoHn Dorrit, The Sleepwalkers. Elucidations of Hermann Broch's Trilogy, The Hague-Paris, Mouton, 1966.

LÜTZeLER Paul Michael, «Erweiterter Naturalismus : Hermann Broch und Emile Zola », Zeitschrift für deutsche Philologie, vol. 93, nº 2, 1974, p. 214-238.

ReINHART Hartmut, Erweiterter Naturalismus. Untersuchungen zum Konstruktionsverfahren in Hermann Brochs Romantrilogie „Die Schlafwandler", KölnWien, Böhlau, 1972.

\section{Jakob Julius David}

\section{CEuvres}

David Jakob Julius, Gedichte, Dresden-Leipzig, Minden, 1892.

-, [Nemo], «Assimilation», Dr. Bloch's Oesterreichische Wochenschrift, 7 mai 1897, p. 390-391.

—, «Im Spiegel. Autobiographische Skizze», Das literarische Echo, vol. 4, $\mathrm{n}^{\mathrm{o}} 8$, 1902, p. 528-530.

—, Der Übergang, Berlin, Schuster \& Loeffler, 1903. 
—, Die Zeitung, Frankfurt am Main, Literarische Anstalt Rütten \& Loening, «Die Gesellschaft. Sammlung sozialpsychologischer Monographien, 5 », 1906.

—, "Am Wege sterben», dans Gesammelte Werke, Ernst Heilborn et Erich Schmidt (éd.), München-Leipzig, Piper, 1908, t. IV, p. 103-316.

—, «Der Übergang », dans Gesammelte Werke, Ernst Heilborn et Erich Schmidt (éd.), München-Leipzig, Piper, 1908, t. V, p. 173-400.

-, «Ein Poet?», dans Gesammelte Werke, Ernst Heilborn et Erich Schmidt (éd.), München-Leipzig, Piper, 1908, t. III, p. 152-190.

-, «Franz Grillparzer», dans Gesammelte Werke, Ernst Heilborn et Erich Schmidt (éd.), München-Leipzig, Piper, 1908, t. VII, p. 1-17.

—, "Vom Schaffen und seinen Bedingungen», dans Vom Schaffen. Essays, Jena, Diederichs, 1906, p. 106-166.

—, "Vorwort zu „Ein Regentag“", dans Gesammelte Werke, Ernst Heilborn et Erich Schmidt (éd.), München-Leipzig, Piper, 1908, t. III, p. 193-195.

-, Verstörte Zeit. Erzählungen, Florian Krobb (éd.), Göttingen, Wallstein, 1990.

\section{Critique}

Beutin Wolfgang, "Subversive Potentiale in den Dichtungen Jakob Julius Davids », dans Johann Dvořák (dir.), Radikalismus, demokratische Strömungen und die Moderne in der österreichischen Literatur, Frankfurt am Main et al., P. Lang, "Bremer Beiträge zur Literatur- und Ideengeschichte, 43 », 2003, p. 175-196.

Doll Jürgen, «Mourir en chemin: presse et littérature chez Jakob Julius David (1859-1906)», dans Jacques Le Rider et Renée Wentzig (dir.), Les Journalistes d'Arthur Schnitzler. Satire de la presse et des journalistes dans le théâtre allemand et autrichien contemporain, Tusson, Du Lérot, 1995, p. 103-117.

GAuss Karl-Markus, «Jakob Julius David oder Er starb am Wege», dans Tinte ist bitter. Literarische Porträts aus Barbaropa, Klagenfurt, Wieser, 1992, p. 149-159.

Groeneweg Herman, Jakob Julius David in seinem Verhältnis zur Heimat. Geschichte, Gesellschaft und Literatur, Graz, Stiasny, « Deutsche Quellen und Studien, 4 ", 1929.

Hock Stefan, «David, Jakob Julius», Biographisches Jahrbuch und Deutscher Nekrolog, $\mathrm{n}^{\circ}$ 9, 1908, p. 185-190.

Kraus Karl, «Antworten des Herausgebers », Die Fackel, vol. 8, n 221, 1907, p. 17.

Квовв Florian, «Empfänglichkeit fürs Leid », dans Jakob Julius David, Verstörte Zeit. Erzählungen, Göttingen, Wallstein, 1990, p. 306-331. 
Liessmann Konrad Paul, «Jakob Julius David und die Kunst der Novelle im Fin de siècle», dans Jakob Julius David, Novellen, Salzburg-Wien, Residenz, 1995, p. 259-291.

Реск Clemens, « „Paralysis progressiva.“ Zur Figuration des Bildungsproletariats in Jakob Julius Davids Wien-Roman ,Am Wege sterben "», Internationales Archiv für Sozialgeschichte der deutschen Literatur, vol. $35, \mathrm{n}^{\circ}$ 2, 2010, p. 37-60.

-, «Jakob Julius Davids Naturalismen», dans Roland Innerhofer et Daniela Strigl (dir.), Sonderweg in Schwarzgelb? Auf der Suche nach einem österreichischen Naturalismus in der Literatur, Innsbruck, Studienverlag, 2016, p. 153-168.

Schamann Franz, «Aus Jakob Julius Davids Nachlaß», Österreichische Rundschau, $\mathrm{n}^{\circ} 23,1910$, p. 429.

—, «Jakob Julius David», Arbeiter-Zeitung, 14 avril 1908, p. 1-3.

Scheuer Oskar F., «Der Wiener Student im Roman », Neues Wiener Tagblatt, 19 mai 1926, p. 1-4.

Schмidт Erich, "Vorwort», dans Jakob Julius David, Gesammelte Werke, Ernst Heilborn et Erich Schmidt (éd.), München-Leipzig, Piper, 1908, t. I, p. V-XXIII.

Spiero Ella, Jakob Julius David, Leipzig, Finck, 1920.

Tateo Giovanni, «Zwischen Hauptstadt und mährischer Provinz. Jakob Julius Davids Erzählung „Die Hanna“”, Studia Germanica Posnaniensia, n 32, 2011, p. 121-135.

Werner Richard Maria, «Jakob Julius David. Ein Erinnerungsblatt», Westermanns Monatshefte, vol. 55, $\mathrm{n}^{\circ}$ 4, 1910, p. 343.

Zweig Stefan, «Dem Gedächtnis Jakob Julius Davids», Österreichische Rundschau, $\mathrm{n}^{\circ}$ 9, 1906, p. 217-219.

\section{Marie von Ebner-Eschenbach}

\section{CEuvres}

Ebner-Eschenbach Marie von, Das Gemeindekind. Novellen, Aphorismen. München, Winkler 1956.

-, Aus Franzensbad. Das Gemeindekind, Evelyne Polt-Heinzl und Ulrike Tanzer (éd.), Ulrike Tanzer (préf.), St. Pölten-Salzburg-Wien, Residenz, «Leseausgabe in vier Bänden, 1 », 2014, p. 127-347.

-, Tagebücher III. 1879-1889. Kritische Texte und Deutungen, Karl Konrad Polheim et Norbert Gabriel (éd.), Tübingen, Niemeyer, 1993.

-, Tagebücher VI. 1906-1916. Kritische Texte und Deutungen, Karl Konrad Polheim et Norbert Gabriel (éd.), Tübingen, Niemeyer, 1997. 
- et Josephine von Knorr, Briefwechsel 1851-1908. Kritische und kommentierte Ausgabe. Mit Marie von Ebner-Eschenbachs historischer Studie Carl I. von England und die hervorragenden Charactere seiner Zeit (1854), Ulrike Tanzer et al. (éd.), Berlin-Boston, De Gruyter, 2016, 2 vol.

\section{Critique}

Anton Christine, «Marie von Ebner-Eschenbach und die Realismusdebatte. Schreiben als Auseinandersetzung mit den Kunstansichten ihrer Zeit ", Modern Austrian Literature, vol. 33, $\mathrm{n}^{\circ}$ 1, 2000, p. 1-15.

Alkemade Mechthild, Die Lebens- und Weltanschauung der Freifrau Marie von Ebner-Eschenbach. Mit 6 Tafelbeilagen und dem Briefwechsel Heyse und Ebner-Eschenbach, Graz-Würzburg, Wächter, 1935.

Aust Hugo, "Am Rande des Realismus : Marie von Ebner-Eschenbachs späte Ehegeschichten aus Dorf und Schloss», dans Claudia Meyer (dir.), Bis zum Lorbeer versteig ich mich nicht. Festschrift für Jürgen Hein, Münster, Ardey, 2007, p. 225-235.

Bettelheim Anton, Marie von Ebner-Eschenbach: biographische Blätter, Berlin, Paetel, 1900.

Hubmann Philipp, “Ich, dem's alle Tage geschehen kann, er weiß nicht wie, daß er Einen erschlagen muß.“ Biopolitik und Risikobewusstsein in Marie von Ebner-Eschenbachs Das Gemeindekind ", dans Thomas Wegmann und Martina King (dir.), Fallgeschichte(n) als Narrativ zwischen Literatur und Wissen. Innsbruck, Innsbruck University Press, "Germanistische Reihe, 84 ", 2016, p. 167-193.

Pfeiffer Peter C., Marie von Ebner-Eschenbach. Tragödie, Erzählung, Heimatfilm, Tübingen, Narr-Francke, 2008.

Rossbacher Karlheinz, «Marie von Ebner-Eschenbach. Zum Verhältnis von Literatur und Sozialgeschichte am Beispiel von Krambambuli», Österreich in Geschichte und Literatur, vol. 24, $\mathrm{n}^{\mathrm{o}}$ 2, 1980, p. 87-106.

Strigl Daniela, Berühmt sein ist nichts. Marie von Ebner-Eschenbach. Eine Biographie, Salzburg-Wien, Residenz, 2016.

\section{Ludwig von Ficker}

\section{CEuvres}

FICKER Ludwig von, Und Friede den Menschen! Eine Christnachtstragödie, Linz, Oesterreichische Verlagsanstalt, 1901.

—, «Franz Schamann : Mährische Geschichten», Tiroler Anzeiger, n ${ }^{\circ}$ 112, 1902. 
-, Inbrunst des Sturms. Ein Reigen Verse, Leipzig-Berlin, Modernes Verlagsbureau Curt Wigand, 1904.

-, Denkzettel und Danksagungen: Aufsätze, Reden, Franz Seyr (éd.), München, Kösel, 1967.

—, Briefwechsel 1909-1914, Ignaz Zangerle et al. (éd.), Salzburg, Otto Müller, 1986.

\section{Critique}

SCHeichl Sigurd Paul, «Aspekte des Judentums im ,Brenner (1910-1937)», dans Walter Methlagl et al. (dir.), Untersuchungen zum ,Brenner'. Festschrift für Ignaz Zangerle zum 75. Geburtstag, Salzburg, Otto Müller, 1981.

Unterkircher Anton, "Ludwig von Ficker», dans Zeitmesser. 100 Jahre ,Brenner, Forschungsinstitut Brenner-Archiv der Universität Innsbruck, Innsbruck, IUP, 2010.

\section{Norbert Gstrein}

CEuvre

Gstrein Norbert, Einer, Frankfurt am Main, Suhrkamp, 1992.

\section{Critique}

Gunreben Marie, Der Literatur mit ihren eigenen Mitteln entkommen. Norbert Gstreins Poetik der Skepsis, Bamberg, University of Bamberg Press, 2011.

\section{Else Jerusalem}

\section{CEuvre}

Jerusalem Else [Kotanyi, Else], Venus am Kreuz. Drei Novellen, Leipzig-Berlin, Meyer, 1899.

-, Gebt uns die Wahrheit! Ein Beitrag zu unserer Erziehung zur Ehe, Leipzig, Seemann, 1902.

—, Der heilige Skarabäus. Roman, [1909], Brigitte Spreitzer (éd.), Wien, dvb. Das vergessene Buch, 2016. 


\section{Karl Kraus}

\section{CEuvres}

Kraus Karl, «Zur Überwindung des Hermann Bahr», dans Frühe Schriften, vol. 1 : 1892-1896, Johannes J. Braakenburg (éd.), München, Kösel, 1979, p. 103-114.

—, "Die demolirte Literatur» [texte de la $5^{\mathrm{e}}$ éd., 1899], dans Frühe Schriften, vol. 2 : 1897-1900, Johannes J. Braakenburg (éd.), München, Kösel, 1979, p. $277-297$.

- et WEDEKIND Frank, Briefwechsel 1903-1917, Mirko Nottscheid (éd.), Würzburg, Königshausen \& Neumann, «Wedekind-Lektüren, 5 », 2008.

\section{Critique}

Canetti Elias, "Karl Kraus. École de la résistance», dans La conscience des mots, Roger Lewinter (éd.), Paris, Albin Michel, 1984, p. 51-64.

KnePler Georg, Karl Kraus liest Offenbach. Erinnerungen. Kommentare. Dokumentationen, Wien, Löcker, 1984.

KRAFT Werner, "Gerhart Hauptmann", dans Das Ja des Neinsagers : Karl Kraus und seine geistige Welt, München, edition text + kritik, 1974, p. 147-157.

Timms Edward, Karl Kraus. Satiriker der Apokalypse, Max Looser et Michael Strand (trad.), Wien, Deuticke, 1995 [éd. originale: Karl Kraus, Apocalyptic Satirist, New Haven, Yale University Press, 1986].

ZoHn Harry, "Karl Kraus und der Expressionismus», dans Klaus Amann et Armin Wallas (dir.) Expressionismus in Österreich. Die Literatur und die Künste, Wien-Köln-Weimar, Böhlau, "Literatur in der Geschichte. Geschichte in der Literatur, $30 »$, 1994, p. 515-525.

\section{Hans Lebert}

Braitenhaller Florian, Küss mich, $d u$ Schwein! Hans Leberts diskrete Beziehung zur Moderne, Wien, WUV Universitätsverlag, 2003.

Egyptien Jürgen, «Kreuzfahrten durch den leeren Himmel. Hans Leberts "Wolfshaut" als transzendentales Logbuch", dans Hans Lebert, Die Wolfshaut, Wien et al., Europaverlag, 1993. p. 599-627, postface. 


\section{Ferdinand von Saar}

\section{CEuvre}

SAAR Ferdinand von, Briefwechsel zwischen Ferdinand von Saar und Maria von Ebner-Eschenbach, Heinz Kindermann (éd.). Wien, Wiener Bibliophile Gesellschaft, 1957.

\section{Critique}

TAтEo Giovanni, «„Ein wirklicher Erähler“ : Ferdiannd von Saar und Arthur Schnitzler - ein literarhistorisches Konstrukt? », dans Wolfgang Lukas et Michael Scheffel (dir.), Textschicksale. Das Werk Arthur Schnitzlers im Kontext der Moderne, Berlin-Boston, Walter De Gruyter, 2017, p. 41-57.

\section{George Saiko}

\section{CEuvre}

SaIko George, Der Mann im Schilf. Roman, dans Sämtliche Werke in fünf Bänden, Adolf Haslinger (éd.), Salzburg-Wien, Residenz, 1985, t. II.

-, Drama und Essays, dans Sämtliche Werke in fünf Bänden, Adolf Haslinger (éd.), Salzburg-Wien, Residenz, 1986, t. IV.

\section{Critique}

MüLler Sabine, «Unbehagliche Perspektiven. Masse, Recht und Führerschaft in George Saikos Roman „Der Mann im Schilf" », dans «Österreichische Literatur ", Treibhaus. Jahrbuch für die Literatur der fünfziger Jahre, vol. 10, 2014, p. 134-158.

\section{Arthur Schnitzler}

\section{CEuvre}

Schnitzlen Arthur, Romans et nouvelles, Brigitte Vergne-Cain et Gérard Rudent (éd.), vol. 1: 1885-1908; vol. 2 : 1909-1931, Paris, Librairie générale française, «Le Livre de poche. Pochothèque », 1994-1996.

-, Frau Berta Garlan [1901], Konstanze Fliedl (éd.), Stuttgart, Philipp Reclam, 2006.

-, Sterben [1894], Hee-Ju Kim (éd.), Stuttgart, Philipp Reclam, 2006.

-, Therese. Chronik eines Frauenlebens [1928], München, dtv, 2008. 


\section{Critique}

Aurnhammer Achim (éd.), Arhur Schnitzlers Lektüren : Leseliste und virtuelle Bibliothek, Würzburg, Ergon, "Akten des Arthur Schnitzler-Archivs der Universität Freiburg, $2 », 2013$.

CRETON Laurence, «De Vienne au crépuscule à Thérèse : les métamorphoses du mythe urbain », dans Rolf Wintermeyer et Karl Zieger (dir.), Les Jeunes Viennois ont pris de l'âge, Valenciennes, Presses universitaires de Valenciennes, 2004, p. 79-9o.

Dangel-Pelloquin Elsbeth, Wiederholung als Schicksal. Arthur Schnitzlers Roman "Therese. Chronik eines Frauenlebens », München, Fink, 1985.

-, «Frau Berta Garlan. Unvermutete Gefühle - ratloses Staunen », dans HeeJu Kim et Günter Saße (dir.), Interpretationen : Arthur Schntzler. Dramen und Erzählungen, Stuttgart, Philipp Reclam jun., 2007, p. 89-100.

FLIEDL Konstanze, Arthur Schnitzler, Stuttgart, Philipp Reclam jun., 2005

JÜRGENSEN Christoph et al. (dir.), Schnitzler Handbuch. Leben - Werk Wirkung, Stuttgart, Weimar, J. B. Metzler, 2014.

Косн-Didier Adelheid, " "Gegen gewisse sozusagen mystische Tendenzen". L'œuvre romanesque d'Arthur Schnitzler », Austriaca, nº 50, 2000, p. 89-107.

Le Rider Jacques, Arthur Schnitzler ou la Belle Époque viennoise, Paris, Belin, "Voix allemandes», 2003.

Pietzker Carl, «Sterben. Eine nouvelle expérimentale », dans Hee-Ju Kim et Günter Saße (dir.), Interpretationen : Arthur Schntzler. Dramen und Erzählungen, Stuttgart, Philipp Reclam jun., 2007, p. 31-45.

ScheIchl Sigurd Paul, «Échos tardifs du naturalisme: Mademoiselle Else et autres récits tardifs d'Arthur Schnitzler », dans R. Wintermeyer et K. Zieger (dir.), Les Jeunes Viennois..., op. cit., p. 91-100.

Zieger Karl, Arthur Schnitzler et la France 1894-1938. Enquête sur une réception, Villeneuve-d'Ascq, Presses universitaires du Septentrion, 2012.

-, «Arthur Schnitzler et le naturalisme: Das Märchen à l'épreuve de la dramaturgie naturaliste », dans Franck Bauer et Guy Ducrey (dir.), Le théâtre incarné. Études en hommage à Monique Dubar, Lille, UL3, "Travaux et recherches", 2003, p. 65-76.

-, «Le naturalisme en Autriche: de la réception de l'œuvre de Zola à la face cachée de "Vienne fin-de-siècle" ", Excavatio, vol. 26, 2015.

-, «Arthur Schnitzler und der Naturalismus : Der naturalistische Einfluss auf seine frühen Dramen », dans Wolfgang Lukas et Michael Scheffel (dir.), Textschicksale. Das Werk Arthur Schnitzlers im Kontext der Moderne, BerlinBoston, Walter De Gruyter, 2017, p. 27-40. 
Josef Winkler

CEuvre

WinkLer Josef, Der Ackermann aus Kärnten. Roman, Frankfurt am Main, Suhrkamp, 1984.

\section{Critique}

UjMA Christina, «Barocker Naturalismus. Josef Winklers römische Novelle „Natura Morta“", literaturkritik.de, no 4, avril 2002.

\section{Émile Zola}

ZIEGER Karl, Die Aufnahme der Werke von Émile Zola durch die österreichische Literaturkritik der Jahrhundertwende, Bern et al., Peter Lang, «Europäische Hochschulschriften : Reihe 18, Vergleichende Literaturwissenschaft, 44 ", 1986.

-, «Die Rezeption der Rougon-Macquart in der österreichischen Arbeiterschaft zwischen der Jahrhundertwende und dem Ständestaat 1934", dans Winfried Engler et Rita Schober (dir.), 100 Jahre Rougon-Macquart im Wandel der Rezeptionsgeschichte, Tübingen, Narr, 1995, p. 195-208.

\section{CEuvres de fiction (en dehors des auteurs et des œuvres cités précédemment)}

Anonyme [Felix Salten], Josefine Mutzenbacher, oder Die Geschichte einer Wienerischen Dirne von ihr selbst erzählt [1906], München, Schneekluth, 1990 [Josefine Mutzenbacher. Histoire d'une fille de Vienne racontée par elle-même, Paris, Mercure de France, 1979; rééd. : Paris, Gallimard «Folio», 1998].

Adamus Franz, Familie Wawroch. Ein österreichisches Drama, Paris-LeipzigMünchen, A. Langen, 1899.

-, Jahrhundertwende. Ein Dramencyklus, Friedbert Aspetsberger (éd.), Innsbruck, StudienVerlag, 2008.

Bettauer Hugo, Die freudlose Gasse. Ein Wiener Roman aus unseren Tagen, Wien-Leipzig, Löwit, 1924.

Böнme Margarete, Tagebuch einer Verlorenen, von einer Toten, Berlin, Fontane, 1905. 
Holmsen Bjarne P. [Arno Holz und Johannes Schlaf], Papa Hamlet, Leipzig, Reißner, 1889.

Langmann Philipp, Bartel Turaser, Leipzig, Friese, 1898.

—, «Ein Unfall», Moderne Rundschau, $\mathrm{n}^{\circ}$ 2, 1891, p. 298-302.

—, Arbeiterleben! Sechs Novellen, Leipzig, Friedrich, s. d. [1893].

Ibsen Henrik, Gespenster. Ein Volksfeind, H. Gimmler (trad.), Nördlingen, Greno, 1989.

\section{Histoire et critique littéraires (ouvrages cités)}

Aspetsberger Friedbert (dir.), Traditionen in der neueren österreichischen Literatur, Wien, Österreichischer Bundesverlag, 1980.

-, Arnolt Bronnen, Wien, Böhlau, 1995.

Amann Klaus, Der Zweite Weltkrieg in der Literatur. Österreichische Beispiele, Bregenz, Erinnern.at, 2002.

Bachleitner Norbert, "Le Théâtre libre de Viene», dans Philippe Baron (dir.), Le Théâtre libre d'Antoine et les théâtres de recherche étrangers, Paris, L'Harmattan, "Univers théâtral », 2007, p. 39-54.

- et al. (dir.), Zola en Europe centrale, Valenciennes, Presses universitaires de Valenciennes, «Europe(s)», 2011.

Barjonet Aurélie, " "Le mieux est de rester volé et content." Armin Schwarz et Gustav Grimm, pirates des œuvres de Zola », Cahiers naturalistes, $\mathrm{n}^{\circ} 81$, 2007, p. 181-196.

BAssler Moritz, Deutsche Erzählprosa 1850-1950. Eine Geschichte literarischer Verfahren, Berlin, Erich Schmidt, 2015.

Becker Colette et Dufief Pierre-Jean (dir.), Dictionnaire des naturalismes, Paris, Champion, 2017 (2 vol.).

Becker Sabina, Neue Sachlichkeit. vol. 1 : Ästhetik der neusachlichen Literatur (1920-1933), vol. 2 : Quellen und Dokumente, Köln-Weimar-Wien, Böhlau 2000.

Benjamin Walter, «Die Wiederkehr des Flaneurs » [1929], dans Gesammelte Schriften, Hella Tiedermann-Bartels (éd.), Frankfurt am Main, Suhrkamp, 1972, t. III, p. 194-199.

BIAsi Pierre-Marc de, Gustave Flaubert. Une manière spéciale de vivre, Paris, Grasset 2009.

BiнA Otto, «Der proletarische Massenroman. Eine neue Eine-Mark-Serie des "Internationalen Arbeiterverlages“" [1930], dans A. Kaes (éd.), Weimarer Republik. Manifeste und Dokumente zur deutschen Literatur 1918-1933, Stuttgart, Metzler, 1983, p. 472-474. 
Bogdal Klaus Michael, Schaurige Bilder. Der Arbeiter im Blick des Bürgers, Frankfurt am Main, Syndikat, 1978.

-, Historische Diskursanalyse der Literatur. Theorie, Arbeitsfelder, Analysen, Vermittlung, Opladen, Westdeutscher Verlag, 1999.

Brauneck Manfred et Müller Christine (dir.), Naturalismus. Manifeste und Dokumente zur deutschen Literatur 1880-1900, Stuttgart, Metzler, 1987.

Bunzel Wolfgang, Einführung in die Literatur des Naturalismus, Darmstadt, Wissenschaftliche Buchgesellschaft, 2008.

Charle Christophe, Le siècle de la presse (1830-1939), Paris, Seuil, «L'Univers historique $", 2004$.

Chevrel Yves, "Naturalisme(s) : singulier ou pluriel », dans Colette Becker et Pierre-Jean Dufief, Dictionnaire des naturalismes, Paris, Champion, 2017, t. I, p. 21-33.

—, "Les relations de Zola avec le monde germanique », Les Cahiers naturalistes, $\mathrm{n}^{\mathrm{o}} 46,1973$, p. 227-247.

—, "Germinal et la "révolution littéraire" en Allemagne», Les Cahiers naturalistes, $\mathrm{n}^{\mathrm{o}}$ 50, 1976, p. 146-164.

-, «Réceptions européennes de Zola (1870-1914): quelques éléments d'un dossier complexe », dans Norbert Bachleitner et al. (dir.), Zola en Europe centrale, Valenciennes, Presses universitaires de Valenciennes, 2011, p. 15-35.

Cowen Roy C., Der Naturalismus. Kommentar zu einer Epoche, München, Winkler, 1975.

Csáky Moritz, Das Gedächtnis der Städte. Kulturelle Verflechtungen. Wien und die urbanen Milieus in Zentraleuropa, Wien-Köln-Weimar, Böhlau, 2010.

DiERsch Manfred, Empiriokritizismus und Impressionismus, Berlin, Rütten \& Loening, 1977.

DONNENBERG Josef, «Heimatliteratur in Österreich nach 1945 - rehabilitiert oder antiquiert? », dans Karl Konrad Polheim (dir.), Wesen und Wandel der Heimatliteratur : Am Beispiel der österreichischen Literatur seit 1945, Bern et al., Peter Lang, 1989, p. 39-68.

ERDMANN Ulrich, Vom Naturalismus zum Nationalsozialismus? Zeitgeschichtlichbiographische Studien zu Max Halbe, Gerhart Hauptmann, Johannes Schlaf und Hermann Stehr, Frankfurt am Main et al., Peter Lang, 1997.

FIALA-FüRST Ingeborg, «Was ist „deutschmährische Literatur“? Versuch der Definition eines unselbstverständlichen Objektes », dans Christoph Fackelmann et Wynfrid Kriegleder (dir.), Literatur. Geschichte. Österreich. Probleme, Perspektiven und Bausteine einer österreichischen Literaturgeschichte, Wien-Berlin, LIT, 2011, p. 278-294.

Foucault Michel, Die Ordnung des Diskurses, Frankfurt am Main, Fischer, 1973. 
Gebhard Gunther, Geisler Oliver et Schröter Steffen, "Heimatdenken : Konturen und Konjunkturen. Statt einer Einleitung», dans G. Gebhard, O. Geisler et S. Schröter (dir.), Heimat. Konturen und Konjunkturen eines umstrittenen Konzepts, Bielefeld, transcript Verlag, 2007, p. 9-56.

GöHre Paul, Drei Monate Fabrikarbeiter und Handwerksbursche. Eine praktische Studie, Leipzig, Grunow, 1891.

Greinz Hugo, "Provinzliteratur", Der Kyffhäuser. Deutsche Monatshefte für Kunst und Leben, vol. 1, $\mathrm{n}^{\mathrm{o}}$ 1, 1899, p. 16-17.

Hahnl Hans Heinz, Hofräte. Revoluzzer. Hungerleider. Vierzig verschollene österreichische Literaten, Wien, Atelier, 1990.

Heydemann Klaus, « Jugend auf dem Lande. Zur Tradition des Heimatromans in Österreich », dans Friedbert Aspetsberger (dir.), Traditionen in der neueren österreichischen Literatur, Wien, Österreichischer Bundesverlag, 1980, p. 83-98.

Hoefert Sigfrid, Das Drama des Naturalismus, Stuttgart, Metzler, 1993.

Hofmannsthal Hugo von, Sämtliche Werke. Kritische Ausgabe, Rudolf Hirsch, Anne Bohnenkamp et al. (éd.), t. XXXII : Reden und Aufsätze. 1, Hans-Georg Dewitz, Olivia Varwig et al. (éd.), Frankfurt am Main, S. Fischer, 2015.

-, Briefwechsel mit Marie von Gomperz 1892-1916. Mit Briefen von Nelly von Gomperz, Ulrike Tanzer (éd.), Freiburg in Breisgau, Rombach, « Rombach Wissenschaften ", 2001.

Holek Wenzel, Lebensgang eines deutsch-tschechischen Handarbeiters, Jena, Diederichs, 1909.

InNerhofer Roland et Strigl Daniela (dir.), Sonderweg in Schwarzgelb? Auf der Suche nach einem österreichischen Naturalismus in der Literatur, Innsbruck, StudienVerlag, 2016.

Janich Peter (dir.), Naturalismus und Menschenbild, Hamburg, Meiner, 2008, vol. 1.

KaEs Anton (dir.), Weimarer Republik. Manifeste und Dokumente zur deutschen Literatur 1918-1933, Stuttgart, Metzler, 1983.

KAFITz Dieter, Johannes Schlaf. Weltanschauliche Totalität und Wirklichkeitsblindheit. Ein Beitrag zur Neubestimmung des Naturalismus-Begriffs und zur Herleitung totalitärer Denkformen, Tübingen, Niemeyer 1992.

-, Décadence in Deutschland. Studien zu einem versunkenen Diskurs der 9oer Jahre des 19. Jahrhunderts, Heidelberg, Winter, 2004.

Kalifa Dominique et al. (dir.), La civilisation du journal. Histoire culturelle et littéraire de la presse française au XIX ${ }^{e}$ siècle, Paris, Nouveau Monde, 2011.

Kaufmann Reinhild, Franz Schamann. Eine Monographie, thèse de doctorat, Innsbruck, 1974 . 
Klettenhammer Sieglinde, “ „Der Scirocco ist kein Tiroler Kind und was uns im Brenner vorgesetzt, ist alles eher als Tiroler Art." Die Zeitschrift ,Der Brenner ' 1910-1915", dans Klaus Amann et al. (dir.), Expressionismus in Österreich, Wien et al., Böhlau, 1994.

Korte Hermann, Österreichische Literatur der Gegenwart, Stuttgart, Metzler, 2016.

Krappmann Jörg, Allerhand Übergänge. Interkulturelle Analysen der regionalen Literatur in Böhmen und Mähren sowie der deutschen Literatur in Prag 1890-1918, Bielefeld, transcript, 2013.

—, «Der Rest oder von Toten und Untoten in der österreichischen Literaturgeschichtsschreibung. Ein Drama in fünf Akten », dans Georg Kastner et al. (dir.), Aufder Suche nach Identität. Festschrift für Dieter Anton Binder, Wien, LIT, 2015.

Kriegleder Wynfrid, Eine kurze Geschichte der Literatur in Österreich. Menschen. Bücher. Institutionen, Wien, Praesens, 2011.

Kunne Andrea, Heimat im Roman : Last oder Lust? Transformation eines Genres in der österreichischen Nachkriegsliteratur, Amsterdam, Atlanta, Rodopi, 1991.

-, «Heimatromane Postmodern. Zur Transformation einer Gattung am Beispiel von Reinhard P. Gruber, Gert Jonke und Max Moetz», dans Hubert Orlowski (dir.), Heimat und Heimatliteratur in Vergangenheit und Gegenwart, Poznan, Instytut Filologi Germanskiej Uam, 1993, p. 101-115.

LÄMMERT Eberhard et al. (dir.), Romantheorie. Dokumentation ihrer Geschichte in Deutschland seit 188o, Königstein, Athenäum, 1984.

Landmann Ulrike, Der Anti-Heimatroman - ein österreichisches Phänomen? Zur Entwicklung einer Literaturströmung mit dem Schwerpunkt eines Vergleichs zur Schweizer (Anti-Heimat)Literatur, thèse, Université de Vienne (Philologisch-Kulturwissenschaftliche Fakultät), 2012.

LE Rider Jacques, « Naturalismus in bleu-blanc-rouge, Schwarz-Weiß-Rot und Schwarzgelb ", dans Roland Innerhofer et Daniela Strigl (dir.), Sonderweg in Schwarzgelb? Auf der Suche nach einem österreichischen Naturalismus in der Literatur, Innsbruck, Studienverlag, 2016, p. 33-56.

-, Wien als „Das neue Ghetto“? Arthur Schnitzler und Theodor Herzl im Dialog, Wien, Picus, «Wiener Vorlesungen 171 », 2014.

LoDge David, "The Language of Modernist Fiction: Metaphor and Metonymy», dans Malcolm Bradbury et James McFarlane (dir.), Modernism 1890-1930, Sussex, Harvester Press, 1978, p. 481-496.

-, The Modes of Modern Writing. Metaphor, Metonymy, and the Typology of Modern Literature, London, Arnold 1977.

LoRenz Dagmar, Wiener Moderne [1995], Stuttgart, Metzler, 2007. 
LütZeler Paul Michael, Hermann Broch. Eine Biographie, Frankfurt am Main, Suhrkamp, 1985.

—, "Zur Avantgarde-Diskussion der dreißiger Jahre : Lukács, Broch und Joyce», dans P. M. Lützeler, Zeitgeschichte in Geschichten der Zeit, Bonn, Bouvier, «Studien zur Literatur der Moderne, 15 », 1986, p. 109-140.

Maderthaner Wolfgang, "Von der Zeit um 1860 bis zum Jahr 1945 », dans Peter Csendes et Ferdinand Opll (dir.), Wien. Geschichte einer Stadt. vol. 3 : Von 1790 bis zur Gegenwart, Wien et al., Böhlau, 2006, p. 175-544.

Maнal Günther, Naturalismus, München, Fink, 1975.

MeID Volker (dir.), Geschichte des deutschsprachigen Romans, Stuttgart, Reclam, 2013.

Menasse Robert, Hysterien und andere historische Irrtümer, Rüdiger Wischenbart (postf.), Wien, Sonderzahl, 1996.

Michlen Werner, Darwinismus und Literatur. Naturwissenschaftliche und literarische Intelligenz in Österreich 1859-1914, Wien, Böhlau, 1999.

-, "The Question of Austrian Naturalism», Excavatio, vol. 14, n ${ }^{\circ}$ 1-2, 2001, p. 323-333.

-, «Zur Frage eines österreichischen Naturalismus», dans R. Innerhofer et D. Strigl (dir.), Sonderweg in Schwarzgelb? Auf der Suche nach einem österreichischen Naturalismus in der Literatur, Innsbruck, StudienVerlag, 2016, p. 21-32.

Mıx York-Gothart (dir.), Naturalismus, Fin de Siècle, Expressionismus 1890-1918, München-Wien, Carl Hanser, "Hansers Sozialgeschichte der deutschen Literatur, $7 », 2000$.

Müller-Guttenbrunn Adam, Feuilletons. Erschienen in der Wiener „Deutschen Zeitung" 1886 bis 1892, vol. $2: 1889$ bis 1892, N. Britz (éd.), Wien, Braumüller, 1978, p. 484-494.

Musner Lutz, "Stadt. Masse. Weib. Metropolenwandel, Massenphobie und Misogynie im Fin-de-Siècle », dans Günther Hödl (dir.), Frauen in der Stadt, Linz, Österreichischer Arbeitskreis für Stadtgeschichtsforschung, « Beiträge zur Geschichte der Städte Mitteleuropas, 18 », 2003, p. 63-81.

Nagl Johann W., Zeidler Jakob et Castle Eduard (dir.), Deutsch-österreichische Literaturgeschichte. Ein Handbuch zur Geschichte der deutschen Dichtung in Österreich-Ungarn, Wien, Fromme, 1899-1937, 4 vol.

Polheim Karl Konrad (dir.), Wesen und Wandel der Heimatliteratur: Am Beispiel der österreichischen Literatur seit 1945, Bern et al., Peter Lang, 1989.

Polt-Heinzl Evelyne, Ringstraßenzeit und Wiener Moderne. Porträt einer literarischen Epoche des Übergangs, Wien, Sonderzahl, 2015.

RABENSTEIN-Michel Ingeborg, «Bewältigungsinstrument Antiheimatliteratur», Germanica, $\mathrm{n}^{\mathrm{o}}$ 42. 2008, p. 1-16. 
Rieckmann Jens, Aufbruch in die Moderne. Die Anfänge des Jungen Wien. Österreichische Literatur und Kritik im Fin de Siècle, Frankfurt am Main, Athenäum, 1986.

Rossbacher Karlheinz, Heimatkunstbewegung und Heimatroman. Zu einer Literatursoziologie der Jahrhundertwende. Stuttgart, Klett, «Literaturwissenschaft-Gesellschaftswissenschaft, 13 ", 1975.

-, «Die Provinz, der Bauer und die Heimat in der Literatur des 20. Jahrhunderts », dans J. Kruse et K. Juhl (dir.), Heimat. Referate und Ergebnisse einer Tagung Bad Segeberg, Landeskulturverband Schleswig-Holstein; Bad Segeberg, Evangelische Akademie Nordelbien, 1978, p. 127-151.

-, Literatur und Liberalismus. Zur Kultur der Ringstraßenzeit, Wien, Jugend \& Volk, 1992.

—, «Heimatkunst der frühen Moderne », dans York-Gothart Mix (dir.), Naturalismus, Fin de Siècle, Expressionismus 1890-1918, München-Wien, Carl Hanser, «Hansers Sozialgeschichte der deutschen Literatur, 7 », 2000.

Rothe Norbert (dir.), Naturalismus-Debatte 1891-1896. Dokumente zur Literaturtheorie und Literaturkritik der revolutionären deutschen Sozialdemokratie, Berlin, Akademie, 1986.

SCHEICHL Sigurd Paul, «Bissige Literatur - zahnloser Kanon. Zu Fragen der literarischen Tradition in Österreich », Sprachkunst, $\mathrm{n}^{0}$ 28, 1997, p. 247-274.

—, «Un naturalisme rural. Franz Kranewitter et Karl Schönherr», dans Karl Zieger et Amos Fergombé (dir.), Théâtre naturaliste, théâtre moderne?, Valenciennes, Presses universitaires de Valenciennes, «Études Valenciennoises, 6 », 2001, p. 79-89.

SitTenberger Hans, «Oesterreichische Dialektstücke », Das litterarische Echo. Halbmonatsschrift für Litteraturfreunde, vol. 4, $\mathrm{n}^{\mathrm{o}}$ 8, janvier 1902.

SCHLAwE Fritz, Literarische Zeitschriften 1885-1910, Stuttgart, Metzler, 1965.

ScHмidL Ekkehart, Der Traum vom Volkstheater. Die Geschichte der Exl-Bühne (1902-1956), Innsbruck, Haymon, «Veröffentlichungen des Innsbrucker Stadtarchivs N. F., $49 », 2013$.

Schmitz Hermann, Jenseits des Naturalismus, Freiburg in Breisgau et al., Alber, «Neue Phänomenologie, 14 », 2010.

Scнütz Erhard et Vogt Jochen, Einführung in die deutsche Literatur des 20. Jahrhunderts. vol. 1 : Kaiserreich, Opladen, Westdeutscher Verlag, 1977.

SONNLEITNER Johann, "Ludwig Anzengruber - Naturalist post mortem? », dans R. Innerhofer et D. Strigl (dir.), Sonderweg in Schwarzgelb? Auf der Suche nach einem österreichischen Naturalismus in der Literatur, Innsbruck, StudienVerlag, 2016, p. 126-138.

SPRENGel Peter, Darwin in der Poesie. Spuren der Evolutionslehre in der deutschsprachigen Literatur des 19. und 20. Jahrhunderts, Würzburg, Königshausen \& Neumann, 1998. 
—, Geschichte der deutschsprachigen Literatur 1870-1900, München, Beck, 1998.

- et Streim Gregor, Berliner und Wiener Moderne. Vermittlungen und Abgrenzungen in Literatur, Theater, Publizistik, Wien, Böhlau, "Literatur in der Geschichte, Geschichte in der Literatur, 45 », 1998.

STEINECKE Hartmut, «, Verwandlungskünstler'? Zur Literaturkritik des Jungen Wien », dans Benjamin Bennett et al. (dir.), Probleme der Moderne. Studien zur deutschen Literatur von Nietzsche bis Brecht. Festschrift für Walter Sokel, Tübingen, Max Niemeyer Verlag, 1983, p. 101-116.

STEINer Rudolf, “ „Bartel Turaser“. Drama in drei Akten von Philipp Langmann » [1897], dans Gesammelte Aufsätze zur Dramaturgie 1889-1900, Basel, Rudolf Steiner Stiftung, "Rudolf-Steiner-Gesamtausgabe, 29 ", 2014, p. 245-247.

Stöckmann Ingo, Der Wille zum Willen. Der Naturalismus und die Gründung der literarischen Moderne 1880-1900, Berlin, De Gruyter, 2009.

—, Naturalismus, Stuttgart, Metzler, 2011.

Tateo Giovanni, " „Heute mittag bricht für die deutsche Literatur eine neue Epoche an. "Henrik Ibsens Gespenster im Urteil Theodor Fontanes, Gerhart Hauptmanns und Hermann Bahrs ", Fontane Blätter, nº 90, 2010, p. 86-109.

Tebben Karin (dir.), Deutschsprachige Schriftstellerinnen des Fin de Siècle, Darmstadt, Wissenschaftliche Buchgesellschaft, 1999.

Troll-Borostyáni Irma von, Die Prostitution vor dem Gesetz. Ein Appell ans deutsche Volk und seine Vertreter, Leipzig, Clausner, 1893.

Tunková Jana, Glanz und Narrenglanz. Rudolf Rittner (1869-1943) : Schauspieler, Dramatiker und Kooperationspartner von Otto Brahm und Gerhart Hauptmann, thèse, Olmütz, 2015, dactyl.

VeselÝ Jiř́, "„Es gibt nur eine Kunst und diese ist realistisch!“ Philipp Langmann, der mährische Gerhart Hauptmann », Germanistica pragensia, $\mathrm{n}^{\circ}$ 9, 1984, p. 101-113.

-, «Ebner-Eschenbach - Saar - David. Tschechische Elemente in ihrem Werk und Leben », Lenau Forum, n ${ }^{0}$ 1, 1969, p. 25-45.

Vorderegger Roger, "Regionale Literaturgeschichtsschreibung. Ein Problemaufriss, eine Perspektive», Jahrbuch Franz-Michael-Felder-Archiv der Vorarlberger Landesbibliothek, $\mathrm{n}^{\mathrm{0}}$ 11, 2010, p. 7-19.

Wagner Adolph, Die Ordnung des österreichischen Staatshaushalts, Wien, Gerold, 1863 .

-, Mittel und Wege zur Errettung des deutschen Volkes aus seiner Verarmung. Ein praktischer Beitrag zur Lösung der sozialen Frage, Breslau, Max\&Comp, 1891.

WAGNER Karl (dir.), Moderne Erzähltheorie. Grundlagentexte von Henry James bis zur Gegenwart, Wien, WUV-Universitätsverlag, 2002. 
Weinberg Manfred, «Region, Heimat, Provinz und Literatur(wissenschaft)», dans Sabine Voda Eschgfäller et Milan Horňáček (dir.), Regionalforschung zur Literatur der Moderne, Olmütz, Universitätsverlag, 2012, p. 41-57.

Weininger Otto, Geschlecht und Charakter, Wien, Braumüller, 1902.

WUnBerg Gotthart, «Deutscher Naturalismus und Österreichische Moderne. Thesen zur Wiener Literatur um 1900 ", dans G. Wunberg, Jahrhundertwende. Studien zur Literatur der Moderne, Tübingen, Narr, 2001, p. 187-207.

- (dir.), Die Wiener Moderne. Literatur, Kunst und Musik zwischen 1890 und 1910 [1981], Stuttgart, Reclam, 2000.

- (éd.), Das Junge Wien. Österreichische Literatur- und Kunstkritik 1887-1902, Tübingen, Niemeyer, 1976, 2 vol.

Zeman Herbert (dir.), Literaturgeschichte Österreichs, Freiburg in Breisgau, Rombach, 2014.

-, «Die österreichische Literatur an der Wende vom 19. zum 20. Jahrhundert », dans Literaturgeschichte Österreichs, Freiburg in Breisgau, Rombach, 2014, p. 493-632.

Zeyringer Klaus et Gollner Hellmut, Eine Literaturgeschichte. Österreich seit 1650, Innsbruck, StudienVerlag, 2012.

Zieger Karl et Fergombé Amos (dir.), Théâtre naturaliste, théâtre moderne?, Éléments d'une dramaturgie naturaliste au tournant $d u \mathrm{XIX}^{e}$ au $\mathrm{XX}^{e}$ siècle, Valenciennes, Presses universitaires de Valenciennes, «Études valenciennoises, 6 », 2001.

Zieger Karl et Wintermeyer Rolf (dir.), Les «Jeunes Viennois» ont pris de l'âge. Les cuvres tardives du groupe «Jung Wien» et de leurs contemporains autrichiens, Valenciennes, Presses universitaires de Valenciennes, «Études valenciennoises, $16 », 2004$. 